\title{
Dopant Concentration Measurements by Scanning Force Microscopy via p-n Junctions Stray Fields
}

\author{
Jacopo Dallari and Ugo Valdrè \\ Electron Microscopy Centre, Physics Department of the University and INFM-CNR, via Irnerio 46, \\ 40126 Bologna, Italy
}

(Received March 20; accepted May 11, 1995)

\begin{abstract}
Scanning Force Microscopy, a non-destructive technique, has been applied to p-n junctions to: (i) locate the metallurgical and the electrical junctions; (ii) determine the $z$-component and gradient of the stray electric field; (iii) measure the width of the depletion region on the $\mathrm{n}$ - and p-sides; (iv) work out the dopant concentration in both $\mathrm{n}$ - and p-sides.
\end{abstract}

\section{Introduction}

Semiconductor technology is developing electronic devices with structures in the sub-micrometer region. A technique capable of testing and characterizing these components is therefore necessary. Scanning Probe Microscopy (SPM) [1], and in particular Scanning Force Microscopy (SFM), is a powerful technique for the study of any kind of surface, both of electrical conductors and insulators, and its applications are being extended from the imaging of surface topography to the detection and measure of various other properties, such as electric or magnetic ones. Contactless techniques, such as SPMs are of paramount importance for device analysis because of their high lateral resolution and ability to perform measurements in a non-destructive way.

In fact, the working principle of an SFM is independent on the nature of the interactive force between the sample and the probe carried by the cantilever at its end. The SFM method may be applied both to form topographic images and to detect magnetic and electrostatic forces. When the probe is brought at a relatively small distance (typically 20-200 $\mathrm{nm}$ ) from the specimen surface, the cantilever bends under the action of the external (stray) electric field emanated by a suitable specimen. For a given tip geometry the long range probe/sample interaction controls the lateral resolution. The use of an electrically conducting tip may provide information about the surface potential of semiconductors [2], ferroelectric domains [3], localized charges deposited at the surface of insulators [4] and on the dopant profile in semiconductors [5].

This work reports on some aspects of the investigations on $\mathrm{p}-\mathrm{n}$ junctions by electric force microscopy (EFM), in particular, on the determination of the dopant distribution at the junction. 


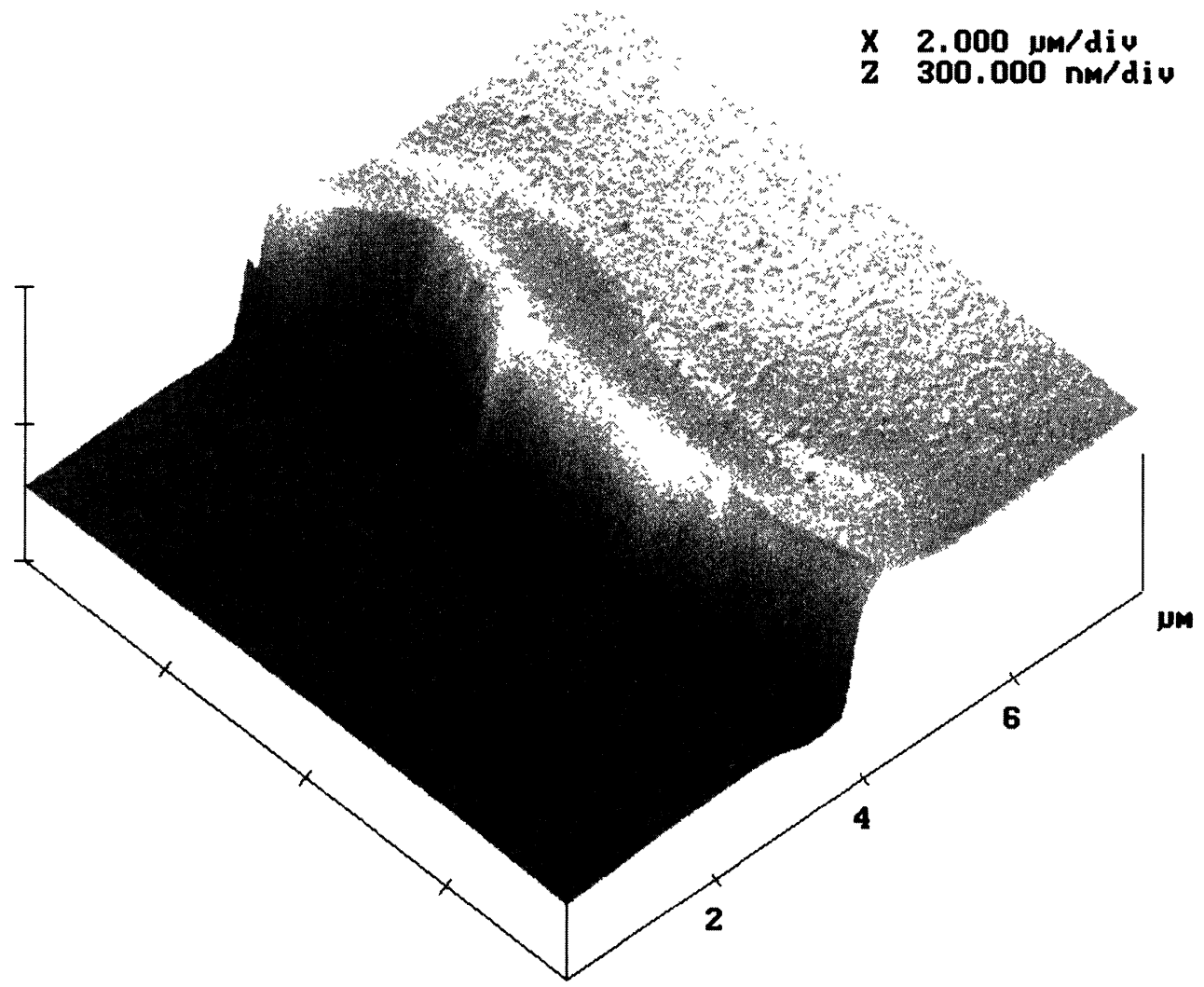

Fig. 1. - Electric force topographic image of the metallurgical junction obtained with a Tapping mode tip. The step's height is about $300 \mathrm{~nm}$.

\section{Instrumentation}

The microscope used is a Digital Nanoscope Multimode III AFM which has been operated in the dynamic (Tapping) mode to achieve better sensitivity to electric fields; in addition, the high spring constant of the Si cantilevers supplied by the manufacturer for the tapping mode renders the cantilever itself less affected by the electrically conducting coating which has to be deposited for EF Microscopy. The coating was obtained by evaporating a gold film, at an angle of about $45^{\circ}$, while the cantilever was rotating. The coating obtained in this way gives good electric performances. The optimum thickness was found to be in the region of 20 to $50 \mathrm{~nm}$. The cantilevers were always used earthed; their resonance frequency was, on the average, $300 \mathrm{kHz}$ and the maximum deflection amplitude about $100 \mathrm{~nm}$.

$\mathrm{A} \pm 12 \mathrm{~V}$ power supply was used to provide a bias voltage to both the $\mathrm{p}$ - and $\mathrm{n}$-regions of the specimen.

All the investigations were performed on commercially available power transistors (Motorola, type $2 \mathrm{~N} 3055$ ). The surface of the devices was usually treated with $50 \% \mathrm{HF}$ to remove the passivating thin oxide layer. Figure 1 shows the topographical step of the metallurgical junction before removal of the $\mathrm{SiO}_{2}$ layer. 


\section{Results}

$3.1 \mathrm{p}-\mathrm{n}$ Junction Detection. - Even at the lowest possible probe/specimen distance (10 nm, dictated by the specimen irregularities) it was not possible to detect any leakage field from an unbiased p-n junction.

By applying a suitable reverse bias voltage $V$ to the junction, it was instead possible to "image" the stray electric field, as can be seen from Figure 2; the tip and the n-region were earthed and a negative voltage was applied to the p-region. The profiles of the electric images clearly show an abrupt change in the proximity of the metallurgical junction. This change is due to the presence of the electrical junction whose distance from the metallurgical one is about $1.5 \mu \mathrm{m}$, in agreement with other work performed in an STEM by the STEBIC technique [6]. In addition, on increasing the reverse bias, both the cantilever deflection and the depletion region increase. The minimum reverse bias voltage which enabled us to discern the presence of the electrical junction is, in the present case, $2.5 \mathrm{~V}$.

3.2 Determination of the $z$-Component of the Stray Electric Field. - The intensity $f_{z}$ and the gradient $f_{z}^{\prime}$ of the $z$-component of the force applied to the cantilever by the stray electric field in any given position (defined by the $x-, y-, z$-coordinates, $z$ being taken perpendicularly to the specimen surface) are obtained by using the following expression [7]:

$$
f_{z}^{\prime}=\frac{3 \sqrt{3}}{2} \frac{\Delta A}{A_{0}} \frac{k_{0}}{Q}
$$

where $k_{0}=54 \mathrm{~N} / \mathrm{m}$ is the spring constant of the free cantilever and $Q=378$ is the quality factor of the resonance curve. $\Delta A$, the change in the oscillation amplitude $A_{0}(=84 \mathrm{~nm})$ of the cantilever, is the experimental datum provided by the microscope.

In order to find the dependence of $f_{z}^{\prime} v s$. $z$ (tip to specimen separation) it has been assumed that the electric field component along $z$ follows an inverse square power law, with $z$ replaced by an effective distance $(z+\beta)$ [8]. Then:

$$
f_{z}^{\prime}=\frac{\alpha}{(z+\beta)^{3}}
$$

where $\alpha$ is a scaling factor. The value of $\beta$ has been determined by applying the leảst square method to equations (1) and (2). It turns out $\beta=65 \mathrm{~nm}$ which is consistent with the value of $A_{0}=84 \mathrm{~nm}$.

In order to work out the dependence of the $z$-component of the electric field on $z$, the following definition equation is used:

$$
E_{z}=\frac{f_{z}}{q(z)}
$$

where $q(z)$ is the probe charge, which depends on the distance tip/sample. $q(z)$ may be found by

$$
q(z)=C(x, z) \cdot V(x)
$$

assuming the configuration depicted in Figure 3, which shows the tip/sample system as three capacitors connected in series [8] of capacitance $C_{\mathrm{air}}, C_{\mathrm{ox}}$ and $C_{\mathrm{d}} . C_{\text {air }}$ is the capacitance of the condensor formed by the tip (assumed to be spherical) and the natural Si oxide with air as dielectric, $C_{\mathrm{ox}}$ is that of the parallel-plate capacitor whose dielectric is the oxide, and $C_{\mathrm{d}}$ refers to 


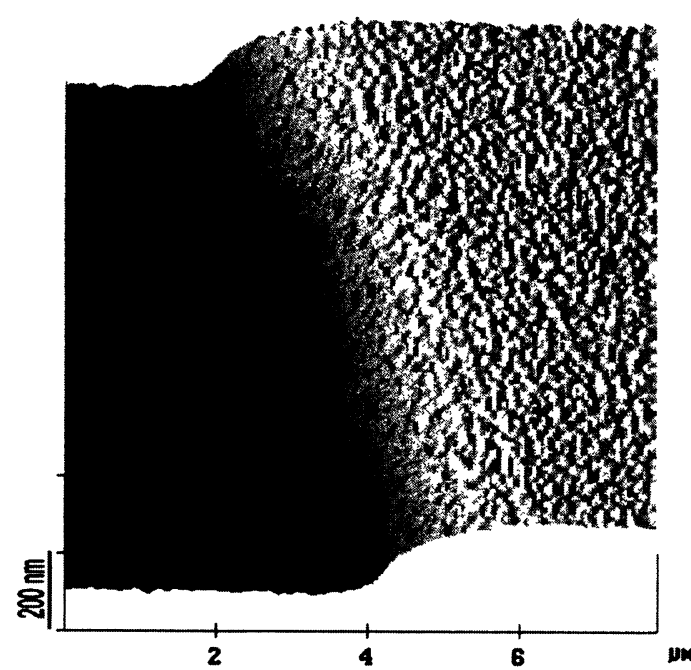

(a)

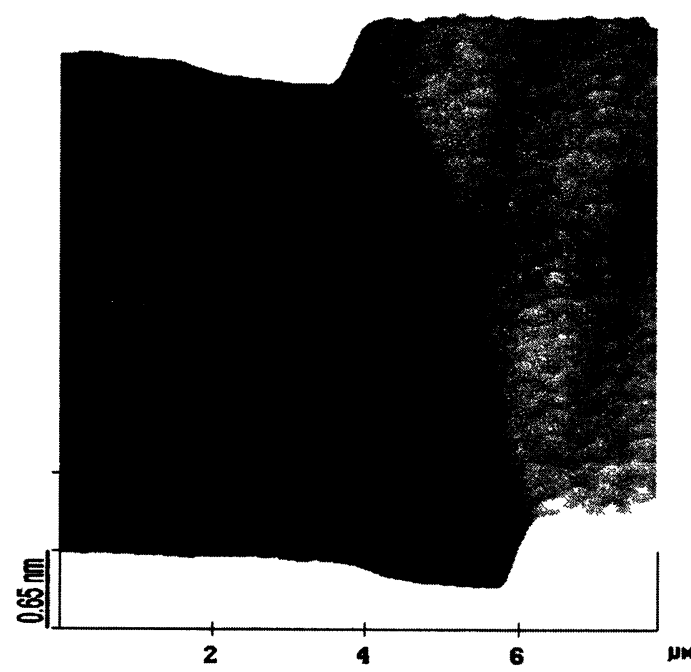

(c)

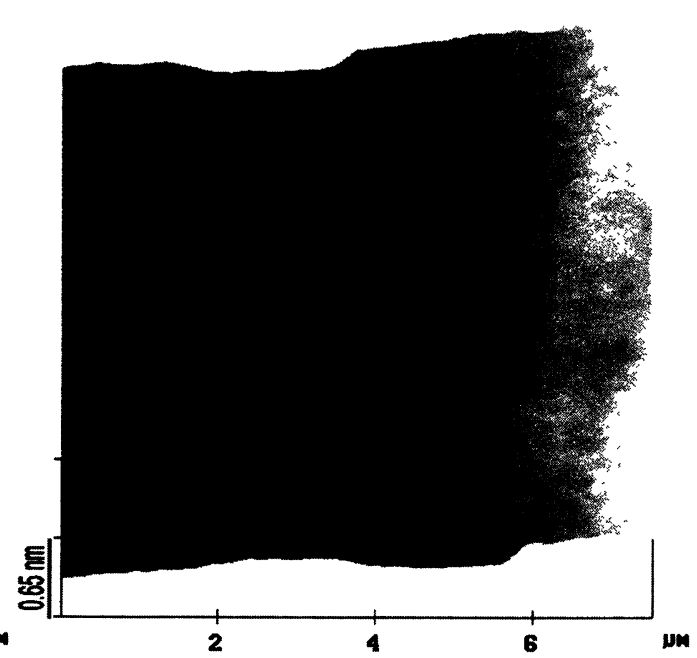

(b)

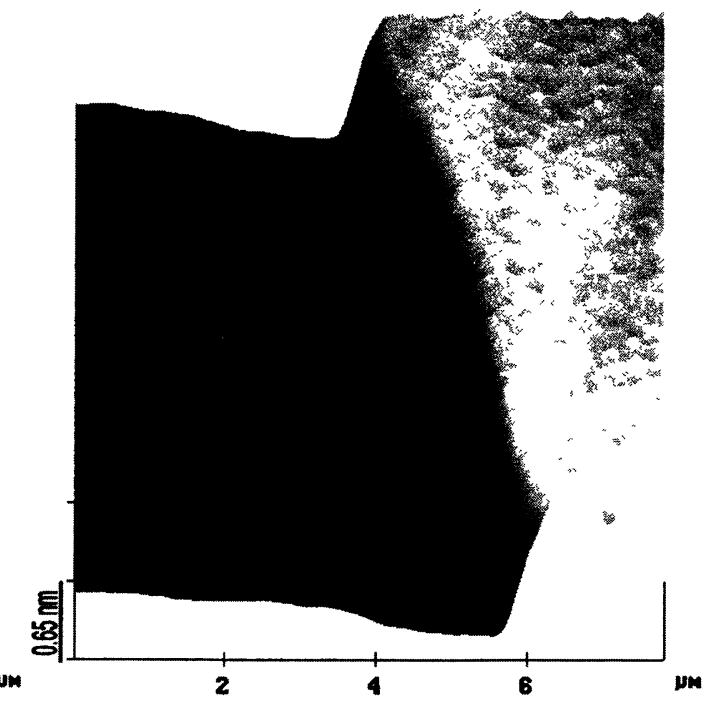

(d)

Fig. 2. - Electron force micrographs of an area of a semiconductor transistor, near a p-n junction, taken with the oscillation (Tapping) mode. (a) Topographic image. Only the metallurgical junction is visible. (b), (c), (d), Set of micrographs of the same area as for (a), recorded at constant distance, for three different reverse bias voltages, $3 \mathrm{~V}, 5 \mathrm{~V}$ and $7 \mathrm{~V}$ respectively. The $\mathrm{n}$-region is earthed and the p-region connected to a negative voltage power supply. The resonance frequency of the cantilever was $298 \mathrm{kHz}$ and the corresponding (maximum) oscillation amplitude $105 \mathrm{~nm}$. The working conditions were chosen at $298.2 \mathrm{kHz}$ and $A_{0}=84 \mathrm{~nm}$. To be noted the sharp change of the electrical signal along a line parallel to, and distant about $1.5 \mu \mathrm{m}$ from, the metallurgical junction, corresponding to the electric junction. 


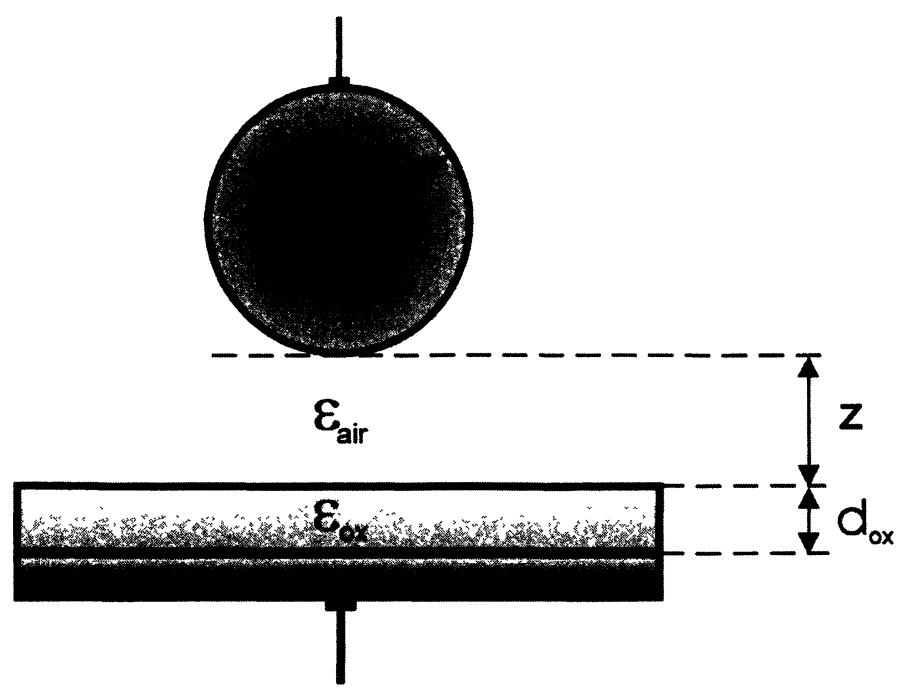

Fig. 3. - Schematic representation of the system tip/transistor as three capacitors connected in series, with dielectric media air, $\mathrm{SiO}_{2}$ and $\mathrm{Si}$.

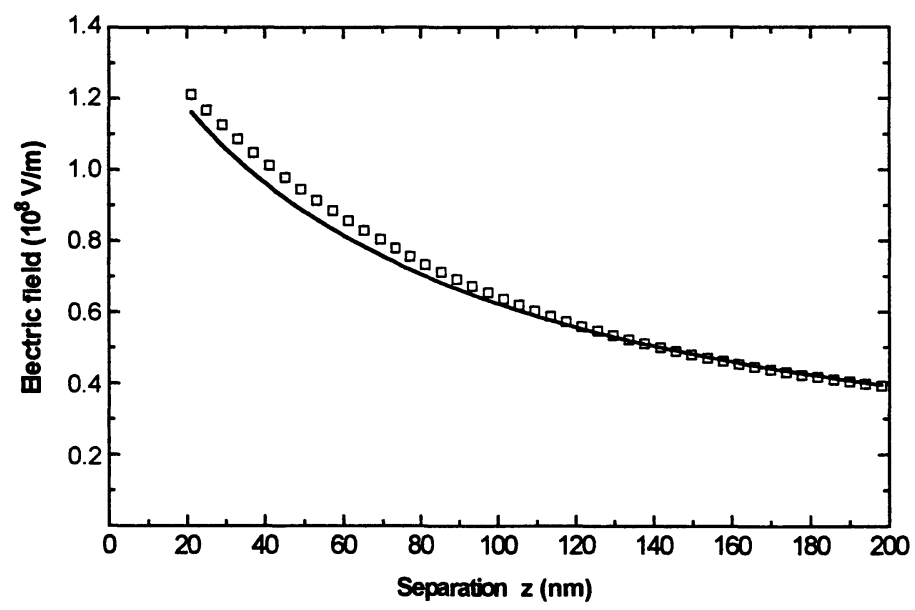

Fig. 4. - Experimentally measured intensity of the $z$-component of the stray electric field above the p-n junction $v s$. the tip/specimen distance $z$. The experimental points are plotted as squares. The continuous line represents the $z^{-1}$ trend. The specimen is a power transistor (Motorola 2N3055) and the applied voltage between $n$ - and p-regions was 0 and $-8 \mathrm{~V}$ respectively. The probe was earthed $(0 \mathrm{~V})$.

the parallel-plate capacitor $\mathrm{SiO}_{2} / \mathrm{Si}$-surface, separated by the semiconductor depletion layer of thickness $t_{\mathrm{d}}$. Thus, the effective capacitance $C$, follows from

$$
\frac{1}{C}=\frac{1}{C_{\mathrm{air}}}+\frac{1}{C_{\mathrm{ox}}}+\frac{1}{C_{\mathrm{d}}}
$$




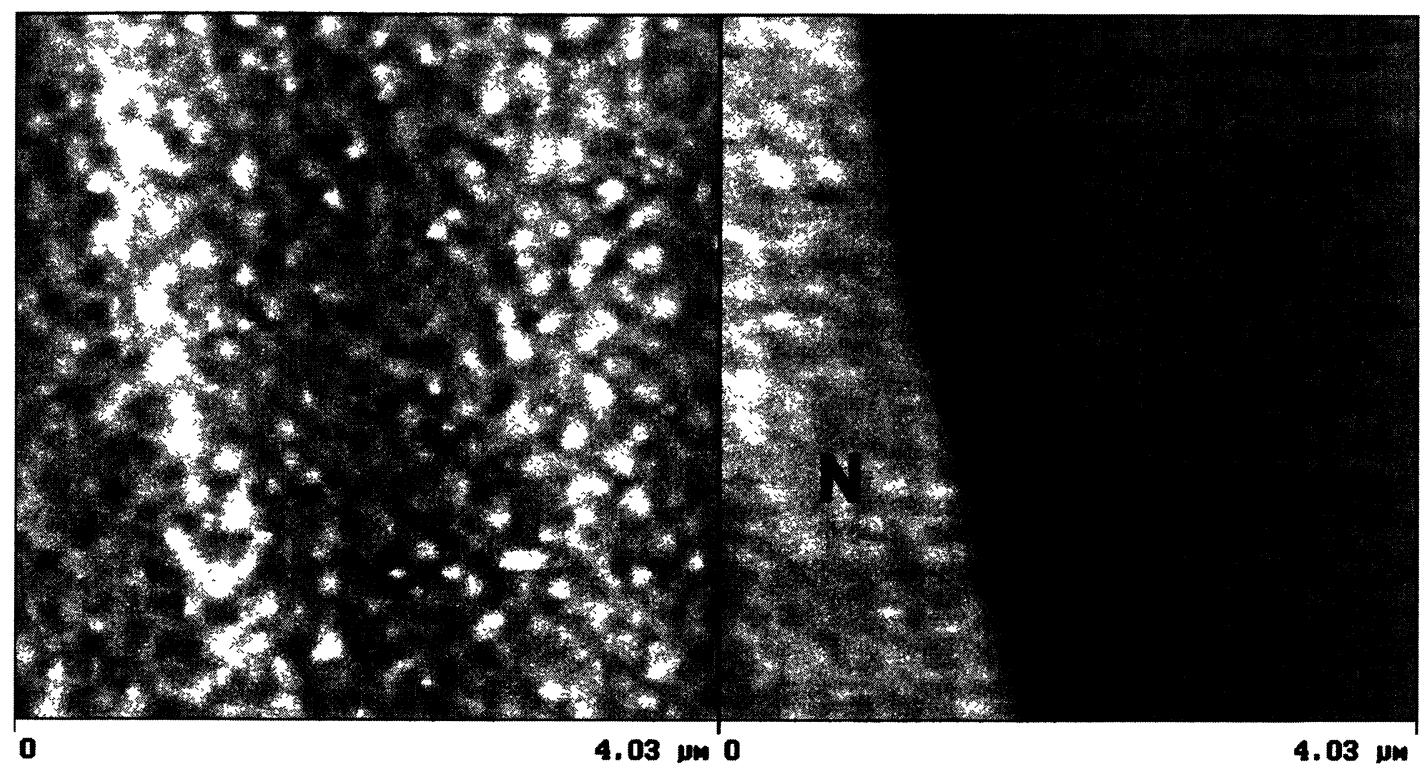

Fig. 5. - Topographic image (left) and electric force image (right) of a region containing a p-n junction. The scanning probe is earthed; a reverse bias voltage of $+4 \mathrm{~V}$ and $-4 \mathrm{~V}$ is applied to the $\mathrm{n}$ - and p-regions respectively. The topographic image (on the left) shows, practically, only an etched surface; the metallurgical junction is located at the left of the micrograph, and excluded from the image. A strong electric contrast is present in the image at the right, which allows an accurate location of the $\mathrm{p} / \mathrm{n}$ transition region.

where

$$
\begin{aligned}
C_{\mathrm{ox}} & =\varepsilon_{\mathrm{ox}} \frac{S}{d_{\mathrm{ox}}}, \\
C_{\mathrm{d}} & =\varepsilon_{\mathrm{d}} \frac{S}{t_{\mathrm{d}}} .
\end{aligned}
$$

$S$ is the "effective" area of the tip given by $2 \pi R^{2}, d_{\mathrm{ox}}$ is the thickness of oxide insulator, and $t_{\mathrm{d}}$ is a function of dc bias $V$ and local dopant volume density [9].

The results are shown in Figure 4. The continuous curve represents the $z^{-1}$ trend, which is in a good agreement with the experimental results (indicated by squares) especially in the high range of $z$.

3.3 Doping Measurements. - Each of the $\Delta A$ electric images (or profiles) of Figure 2 have been obtained with both probe and n-region earthed, and the p-region at a negative voltage. The electric field distribution is therefore expected, as it actually happens, to be strongly asymmetrical. By applying the same reverse bias voltage to the n- and p-regions (but with opposite signs) while keeping the probe earthed, a different electric field distribution is produced, with a deep minimum at the $n / p$ boundary.

In this situation the change of amplitude $\Delta A$ as a function of $x$, for a given $z$, is of the type shown in Figure 6 (squares), which refers to a total reverse bias voltage of $8 \mathrm{~V}$ applied between the nand p-regions. The experimental points drawn in Figure 6 are, in fact, obtained by averaging the line scans which form the image of Figure 5 (right). It is clear that the trend is different from those of Figures $2 \mathrm{~b}, \mathrm{c}, \mathrm{d}$. The depletion region corresponds to the strong change of amplitude, where 


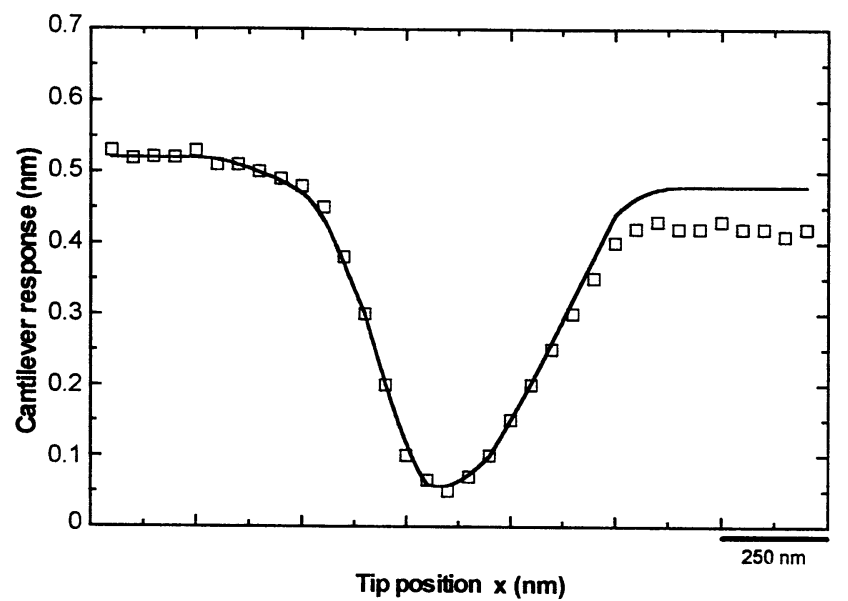

Fig. 6. - The squares represent the experimental amplitude change $\Delta A$ (averaged over $2 \mu \mathrm{m}$ ) of the cantilever as a function of the probe position along a line ( $x$-axis) normal to, and crossing, the p-n junction. The experimental data are taken from Figure 5 . The $\mathrm{p} / \mathrm{n}$ boundary is located at the minimum. The width of the depletion region can be found from this curve. Note the slight asymmetry of the minimum which indicates a difference in the doping level at the two sides of the $\mathrm{p} / \mathrm{n}$ boundary (the $\mathrm{n}$-region is on the left). The continuous line represents the fitted theoretical curve.

the force acting on the cantilever changes rapidly, whereas the two regions outside the depleted region are represented by a constant amplitude. From the experimental curve of Figure 6, slightly asymmetrical, it is possible to measure both the total width, $W=W_{\mathrm{n}}+W_{\mathrm{p}}$ of the depletion region and the widths $W_{\mathrm{n}}$ and $W_{\mathrm{p}}$ corresponding to the $\mathrm{n}$ - and p-regions respectively. The latter values are related to the dopant concentrations in the two regions. The trend of the experimental curve of Figure 6 can be worked out theoretically [9] and the fitting is done by adjusting the values of the dopant concentrations in the $\mathrm{n}$ - and $\mathrm{p}$-regions. The rather good fitting given by the continuous curve in Figure 6 has been obtained by assuming a donor concentration of $3.0 \times 10^{16} \mathrm{~cm}^{-3}$ for the n-region and an acceptor concentration of $2.0 \times 10^{16} \mathrm{~cm}^{-3}$ in the p-region. These values are typical for the type of transistor doubly diffused under investigation. We have estimated that the accuracy of this method is about $10 / 15 \%$ and depends mainly on the evaluation of the position of the boundaries of the depletion region.

\section{Conclusions}

It has been shown and confirmed that the scanning force microscope can be used as a nondestructive method to derive some information about semiconductor devices, such as:

(i) the location of the metallurgical and of the electrical junctions. Truly, the passivating oxide layer, $0.2 \mu \mathrm{m}$ thick, was partly removed from the surface of the device under investigation; however, the sensitivity of the method is such that an oxide layer of the order of $0.1 \mu \mathrm{m}$ can be tolerated. As the miniaturization of the electronic devices proceeds, thinner layers will be used;

(ii) the trend of the derivative of the stray electric field along the $z$-axis and, with the help of a model, of the $z$-component of the stray electric field of a $\mathrm{p}$-n junction. So far a minimum reverse bias voltage of $2.5 \mathrm{~V}$ has been applied in order to increase the detection sensitivity, which helped in setting the technique; however, the results obtained indicate that the intrinsic stray field should be 
detectable in devices presenting a smooth surface (in particular with metallurgical junction heights smaller than $0.1 \mu \mathrm{m}$ ). A practical solution to improve the sensitivity would be the application of a suitable dc voltage to the tip; in this way the interactive force between the sample and the probe increases, thus inducing a greater charge on the tip (see Eq. (4));

(iii) the width of the depletion region on both sides of the $p$ - $n$ junction;

(iv) the dopant concentration in both the p-and n-regions. The latter result is based on an original method, alternative to the usual and conventional methods of resistivity measurements and of electron microscope investigations. The new method has the advantage of being simple, non-destructive and of high resolution. The last two properties are of paramount importance where the size of the semiconductor devices is beyond the capabilities of traditional methods. More work is planned in this area on the examination of various materials and levels of dopant concentration present in commercial devices.

\section{Acknowledgements}

This work has been partly supported by MURST, Rome, and by the European Union.

\section{References}

[1] Binnig G., Quate C.F. and Gerber Ch., Phys. Rev. Lett. 56 (1986) 930.

[2] Martin Y., Abraham D.W. and Wickramasinghe H.K., Appl. Phys. Lett. 52 (1988) 1103.

[3] Lüthi R., Haefke H., Meyer K.P., Meyer E., Howald L. and Güntherodt H.J., J. Appl. Phys. 74 (1993) 7461.

[4] Stern J.E., Terris B.D., Mamin H.J. and Rugar D., Appl. Phys. lett. 53 (1988) 2717.

[5] Abraham D.W., Williams C.C., Slinkman J. and Wickramasinghe H.K., J. Vac. Sci. Technol. B 9 (1991) 703.

[6] Sparrow T.G. and Valdrè U., Philos. Mag. 36 (1977) 1517.

[7] Martin Y., Williams C.C. and Wickramasinghe H.K., J. Appl. Phys. 61 (1987) 4723.

[8] Terris B.D., Stern J.E., Rugar D. and Mamin H.J., Phys. Rev. Lett. 63 (1989) 2669.

[9] Sze S., The Physics of Semiconductor Devices (Wiley, New York, 1981). 